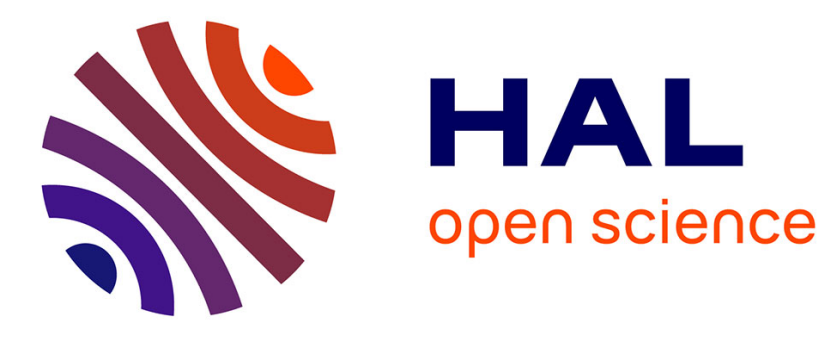

\title{
Metamaterial-like transformed urbanism
}

Stéphane Brulé, Bogdan Ungureanu, Younes Achaoui, Andre Diatta, Ronald Aznavourian, Tryfon Antonakakis, Richard Craster, Stefan Enoch, Sébastien Guenneau

\section{- To cite this version:}

Stéphane Brulé, Bogdan Ungureanu, Younes Achaoui, Andre Diatta, Ronald Aznavourian, et al. Metamaterial-like transformed urbanism. Innovative Infrastructure Solutions, 2017, 2 (1), $10.1007 / \mathrm{s} 41062-017-0063-\mathrm{x}$. hal-01635890

\section{HAL Id: hal-01635890 https://hal.science/hal-01635890}

Submitted on 13 Apr 2018

HAL is a multi-disciplinary open access archive for the deposit and dissemination of scientific research documents, whether they are published or not. The documents may come from teaching and research institutions in France or abroad, or from public or private research centers.
L'archive ouverte pluridisciplinaire HAL, est destinée au dépôt et à la diffusion de documents scientifiques de niveau recherche, publiés ou non, émanant des établissements d'enseignement et de recherche français ou étrangers, des laboratoires publics ou privés. 


\title{
Metamaterial-like transformed urbanism
}

\author{
Stéphane Brûlé ${ }^{1}$ (1) Bogdan Ungureanu ${ }^{1} \cdot$ Younes Achaoui $^{1} \cdot$ André Diatta $^{1} \cdot$ \\ Ronald Aznavourian $^{1}$ - Tryfon Antonakakis ${ }^{2} \cdot$ Richard Craster $^{3} \cdot$ Stefan Enoch $^{1}$. \\ Sébastien Guenneau ${ }^{1}$
}

Received: 9 January 2017/Accepted: 24 April 2017/Published online: 7 June 2017

(C) Springer International Publishing Switzerland 2017

\begin{abstract}
Viewed from the sky, the urban fabric pattern appears similar to the geometry of structured devices called metamaterials; these were developed by Physicists to interact with waves that have wavelengths in the range from nanometers to meters (from electromagnetic to seismic metamaterials). Visionary research in the late 1980s based on the interaction of big cities with seismic signals and more recent studies on seismic metamaterials, made of holes or vertical inclusions in the soil, has generated interest in exploring the multiple interaction effects of seismic waves in the ground and the local resonances of both buried pillars and buildings. Here, we use techniques from transformational optics and theoretically validate, by numerical experiments, that a district of buildings could be considered as a set of above-ground resonators, purely elastic, interacting with an incident seismic signal. We hope that our proposal will contribute to all theoretical and experimental efforts in design of cities of the future, from a metamaterial standpoint.
\end{abstract}

Keywords Metamaterial · Urban fabric - Metacity · Transformational physics $\cdot$ Homogenization

Stéphane Brûlé

stephanebrule@orange.fr

1 CNRS, Centrale Marseille, Institut Fresnel, Aix-Marseille University, Marseille, France

2 Multiwave Technologies AG, Geneva, Switzerland

3 Department of Mathematics, Imperial College London, London, UK

\section{Introduction}

In this short communication, we propose an analysis of the dynamic response of surface structures. These buildings are not studied as single elements with their own characteristics and modeled by a single degree of freedom oscillator (SDOFO) but as an interacting set of above-ground resonators clamped in a common slab, at the scale of a district in a city.

In this article, we analyze a perfect elastic system consisting of soil and buildings. We do not introduce soilfoundation-structure interaction [4] and damping, which are major aspects in earthquake engineering. We simply wish to investigate, given that communities have built highly structured cities, whether the design of a district could benefit from results obtained for waves interacting with structured metamaterials that are built at a smaller scale.

Most of the time in earthquake engineering, the dynamic response of a building is formulated as an elastic response spectrum $S(T, \xi)$, in acceleration $S_{\mathrm{a}}$, velocity $S_{\mathrm{v}}$, or horizontal displacement $S_{\mathrm{d}}$ of an SDOF system (mass $m$, stiffness $k$ ) under seismic acceleration of the soil $\ddot{x}_{g}(t)$. This graph represents all the maximum value of acceleration, velocity, or displacement, for a relative displacement $x(t)$ in time domain, for given damping ratio $\xi$ and a fundamental period $T$ of the structure (Fig. 1a). $x_{T}(t)$ is the overall displacement and $x_{\mathrm{g}}(t)$ is the displacement of the soil recorded at the surface by seismograph.

The novel approach illustrated in this paper suggests to take into account the effects of the overall dynamic response of the system composed of a group of buildings clamped in a common slab (Fig. 1b). The system is not tested under a true seismic disturbance but by varying the frequency step by step. 
Fig. 1 Principle of usual single degree of freedom oscillator model for a building (a). Global approach with a set of resonators modeling a group of buildings of different heights (b) and vibrating in the bending mode in this figure

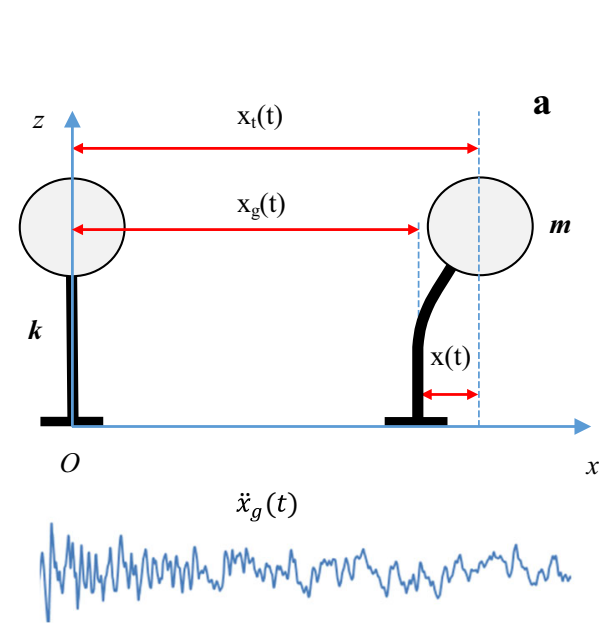

b

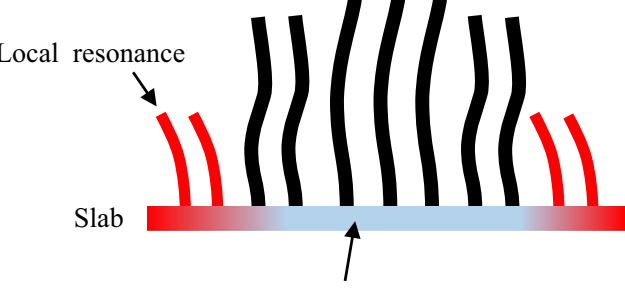

Overall dynamic response of the system
The plan of the paper is as follows: First, we show the similarity of the urban fabric with the geometry of metamaterials ("The concept of metacity" and "From urbanism to metamaterials"), and then, we recall the concept of multi-scale resonant structures and the main characteristics of seismic ground motion ("Earthquakes and resonance structures"). This allows us to propose the concept of a metacity on the basis of resonant structures having their foundations in a common slab ("Meta city and analogy with urban fabric"). We make the assumption of elastic behaviour for each component of the mechanical system (soil, foundation, and buildings). To illustrate the idea of a city district envisioned as a megastructure interacting with the seismic signal, we develop a numerical approach based on finite elements (implemented in the commercial software Comsol Multiphysics). Starting with a square network of streets and buildings, we map it onto a quasi-conformal network of streets with square buildings ("Meta city and analogy with urban fabric"), deduced from some spatially varying (but isotropic) material parameters given by a set of transformed elastodynamic equations. In "Discussion and perspectives", numerical results show Rayleigh waves $(0.5 \mathrm{~Hz})$ detoured around the center of a seismic cloak, which is $1 \mathrm{~km}$ in diameter.

\section{The concept of metacity}

The metacity is a concept first put forward in the late 1980s on the basis of experimental observations of ground response and its devastating effects. It is known, since the $1950 \mathrm{~s}$, that the natural frequencies of any man-made structure are influenced by soil-structure interaction, especially on soft soils $[24,25,50]$, and the presence of structures at the surface of a homogeneous half-space can significantly modify the ground motion [12, 20, 47, 51]. Most of the time, the problem of ground response is disconnected from that of the resonant response of buildings or group of buildings.

On the basis of studies carried out on the interaction of cities with the seismic signal $[2,5,6,21,22]$ and on the interaction of buildings with each other [46], we propose further extensions of this concept (Fig. 2) based on analogies with electromagnetic and seismic metamaterials [11]. Actually, the field of transformational optics, which is 10 years old [41], can help explain metamaterial-like urbanism: When viewed from the sky, some cities look similar to invisibility cloaks. Numerical simulations backup our claim of control of seismic waves via artificial anisotropy in metacities.

The high density of deep foundation or ground reinforcement techniques in the urban area leads researchers to believe that there is a significant interaction of these buried structures with a certain component of the seismic signal. A promising way to cause a modification of the seismic disturbance is to create complete artificial anisotropy by implementing geometrical elements, full or empty, in the soil [11]. The physical process is the interference of waves (body or surface waves) scattered from surfaces or objects. The effects of the anisotropy are reinforced by the local resonance of implemented elements which are placed along a grid according to transformation elastodynamics and morphing tools; these could theoretically lead to an ideal cloak detouring waves around a protected area. In these periodic or non-periodic media, the desired effects are total reflection (Bragg's effect), bandgaps, wave-path control, and mitigation by energy-dissipation, amongst others. 


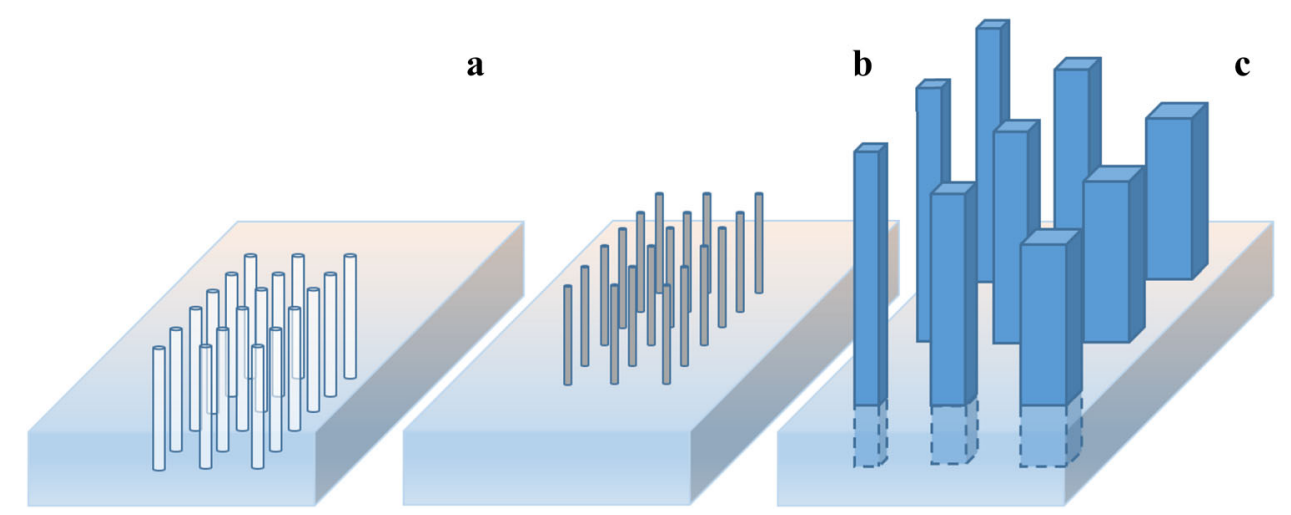

Fig. 2 From left to right (arbitrary scale): a buried columns of deep foundation (piles) or rigid inclusion or holey-ground. b Phononic above-ground structure made of vertical metallic elements, inspired from the sculpture of Eusebio Sempere in Madrid. Meseguer and his

\section{From urbanism to metamaterials}

In urbanism, the urban fabric (Fig. 3) is the physical form of towns and cities and the urban grain defined as the combined pattern of blocks and streets, taking into account the character of street blocks and building height and size, co-authors demonstrated in 1998 the first acoustical band-gap structure in the audible frequency range [38]. Analogy of $\mathbf{b}$ for seismic waves illustrated by a diagram of a neighbourhood with different heights of building and various underground levels (c)

and how they all work together in an interrelated manner to enable movement and access. If we describe in a factual way these structures, observed from the sky, we note a distribution of constructions reminiscent of many manmade structures at different scales, and in particular, metamaterials. An early example, going back in the
Fig. 3 Above illustration of the urban fabric of Paris and New York (sky view). Below plan of the ideal and virtual city of Chaux in France [35]

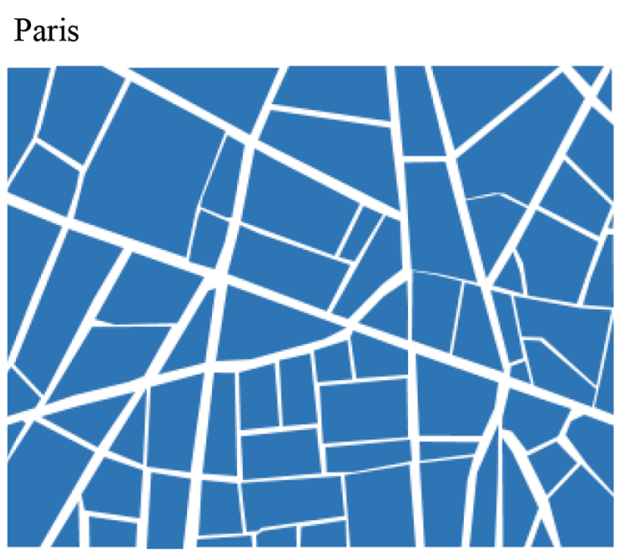

New York

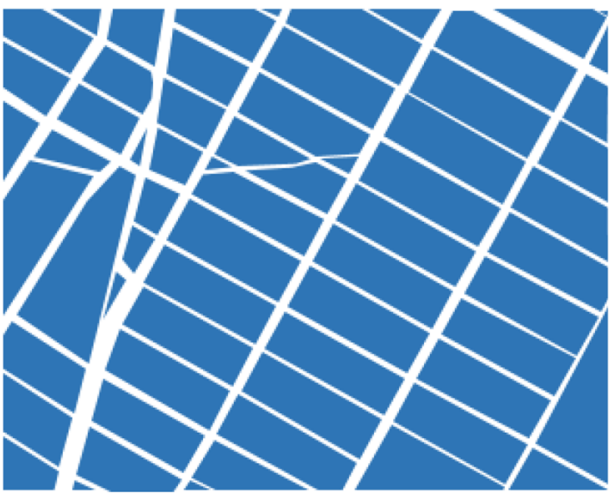

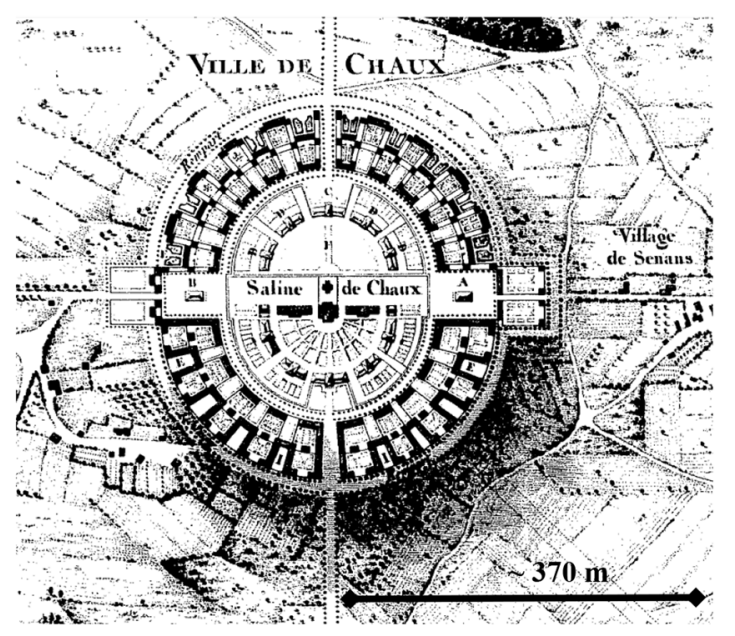




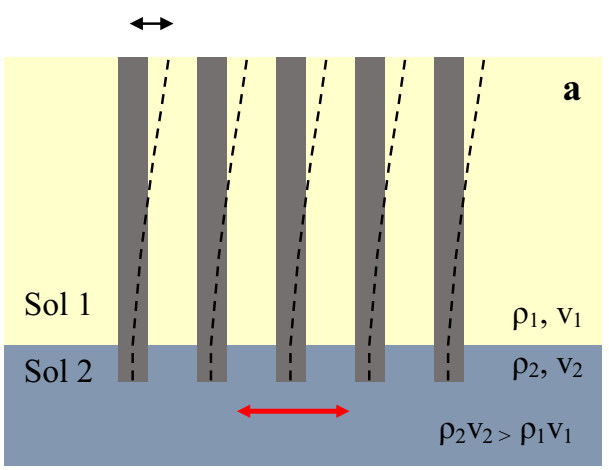

Fig. 4 Schematic view of principle (side view) of vertical columns buried within a soft soil (soil 1) and clamped within a bedrock (soil 2). These columns undergo bending (a) and longitudinal (b) motions under earthquake. These local resonances create elastic stop bands at

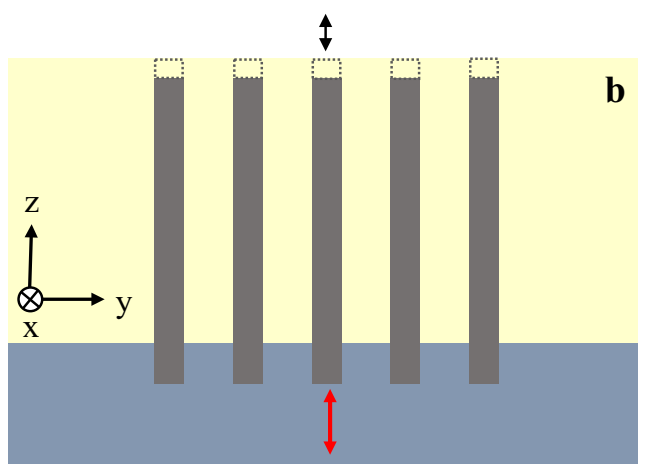

low frequencies (i.e., long wavelengths get reflected by arrays of columns with a deeply subwavelength cross section). Inspired from [10]
Fig. 5 Deformation of building (a). Elastic deformation for the first fundamental mode (b). Ductile deformation (c) and brittle deformation (d)

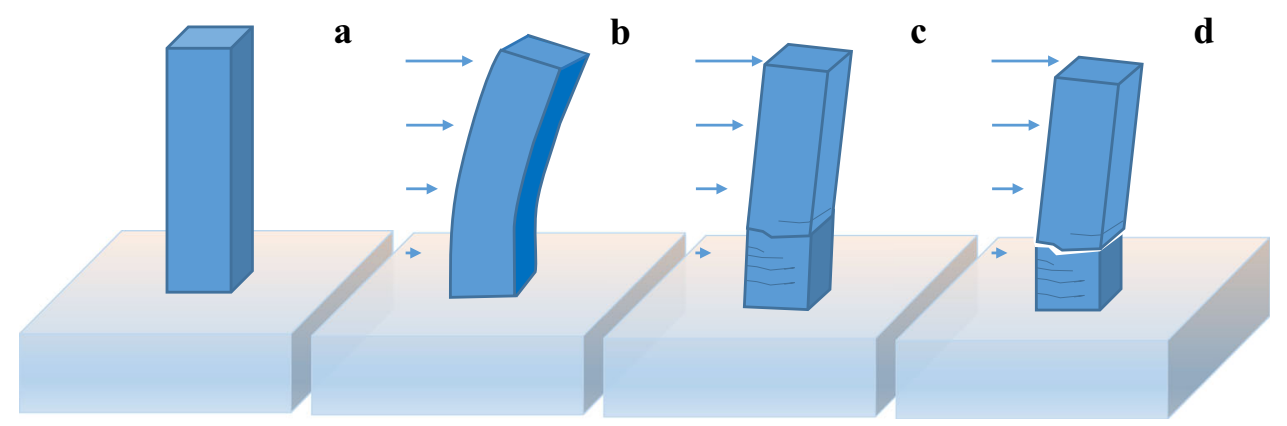

historical record, is the Royal Saltworks (1779) at Arc-etSenans in France, designed and built by the visionary Architect Claude-Nicolas Ledoux (1736-1806); this semicircular complex of buildings (Unesco World Heritage) formed the starting point for his project of a utopian city never built [35].

In 2001, the word "metamaterial" was coined by Walser, who gave the following definition: macroscopic composite having a man-made, three-dimensional, periodic cellular architecture designed to produce an optimized combination, not available in nature, of two or more responses to specific excitation [49]. The concept of metamaterial arose from the nano-scale world and the scientific field of electromagnetism. Typically, a metamaterial uses periodic arrangements of elements with size much smaller than the considered wavelength (typically hundreds of nanometers) that acquire effective properties of materials with negative optical index [40], or highly anisotropic materials such as hyperbolic metamaterials [28] or invisibility cloaking devices [41].

In recent years, one of the objectives of researchers has been to translate these concepts from the nano- to the meter-scale world. This transition from electromagnetic to acoustic and mechanical waves was made possible thanks to phononic crystals, which are artificial man-made structures whose size ranges from a few meters down to hundreds of nanometers or less.

Briefly, the first application suggested by Kushwaha et al. $[32,33]$ was for acoustic insulation for human hearing which is sensitive to frequencies between 20 and 20,000 Hz. In 2005, Hu and Chan [26] proposed a breakwater device for surface ocean waves as a potential application of photonic crystals at the meter-scale domain.

In the same spirit, some of us envisioned in 2008 an approach to reroute ocean waves around a region of still water surrounded by concentric arrays of pillars [18]; nonovertopping dykes for ocean waves can also be envisaged with meter-scale invisibility carpets for water waves [17]. The theoretical concept of a seismic 2D grid of inclusions in the soil interacting with a part of the earthquake signal was translated to reality thanks to full-scale experiments on structured soil by means of empty holes [7, 8, 11, 45]. Researchers have recently been working to improve the concept of structured soil and its effectiveness to counteract the most devastating component of the seismic signal. They act on the densification of the soil itself $[9,10]$ and on the dynamic anisotropy of the medium compounded of soil and vertical rigid elements that could be concrete or steel piles for example (Fig. 4). 


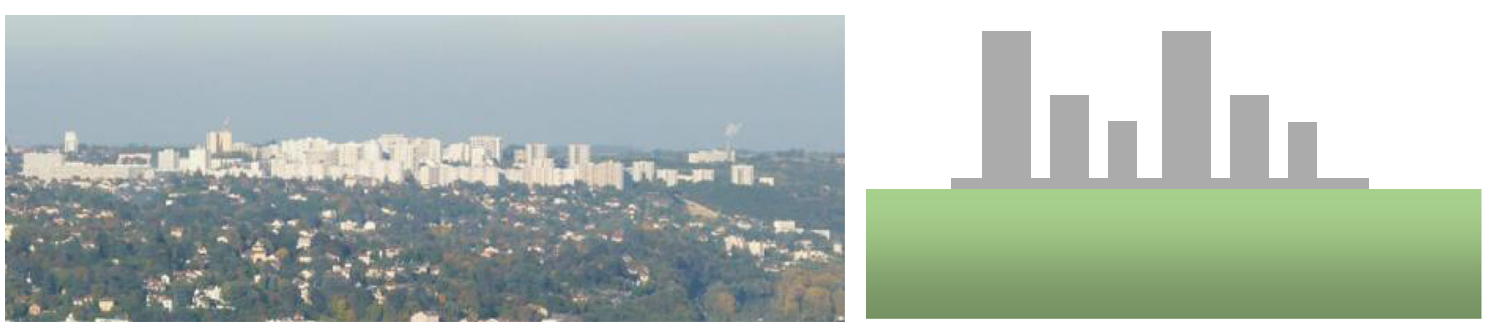

Fig. 6 View of a cluster of buildings (left) suggesting the schematic representation of these buildings clamped on a common concrete slab (right)

The effects of dynamic anisotropy are reinforced by the local resonance of implemented concrete columns, which are placed along a grid according to transformational elastodynamics and morphing tools that could theoretically lead to an ideal cloak detouring waves around a protected area $[3,13,15,16,19]$. Interestingly, forests of trees naturally behave like locally resonant structures, and can shield Rayleigh waves thanks to stop bands above $50 \mathrm{~Hz}$ [14] which is, however, at frequencies higher than those primarily present in earthquakes.

Another way to act on the seismic signal is to investigate the effects of a 3D grid of specifically tuned resonators in the soil $[1,30,31]$. For instance, these resonators could be spheres connected to a bulk of material via ligaments and moving freely within a surrounding air cavity [1]. The objective is to obtain stop bands for seismic waves aiming to avoid resonance of the soil with the main vibration modes of the buildings. Finally, a promising avenue is study using extreme properties materials: For example, auxetic metamaterials, characterized by their negative Poisson's ratio $v$ [34], buried in the soil [48], bring bandgaps at frequencies compatible with seismic waves when they are designed appropriately.

As a reminder, Poisson's ratio is the ratio of transverse contraction strain to longitudinal extension strain in a stretched bar. Since most common materials become thinner in cross section when stretched, their Poisson's ratio is positive and so negative Poisson ratio materials are unusual.

\section{Earthquakes and resonance structures}

Devastating earthquakes are not necessarily located near the faults. Indeed, in the case of the Mexico Earthquake on 19 September 1985, the epicenter was several hundreds of kilometers away from the Mexico City (more than $350 \mathrm{~km}$ ). However, this event caused the loss of 10,000 human lives due, in particular, to damage and settlements on buildings, worsened by so-called "seismic site effects" and induced effects such as soil-liquefaction. Seismic site effects are related to the amplification of seismic waves in superficial geological layers. The surface ground motion may be strongly amplified if the geological conditions are unfavourable (e.g., soft layers of sediments); the latest generation of seismic building codes is based on taking into account these site effects.

We place our study in the case of far-field earthquakes inducing significant ground amplification in alluvium basins. Soils and structures remain in the "small-strain" range that is to say we assume that these materials are strictly in the elastic domain (Fig. 5b).

We do not study the case of the complete change of the topography due to very strong, but rare, earthquakes, i.e., uplift of several meters of the tectonic block (the AD 365 Crete Earthquake, for example).

Until now, and except for particular buildings, earthquake design is rarely a strictly "dynamic problem". Indeed, the dynamic seismic motion is converted into an equivalent static load applied to the structure. This remains a rather simple approach and the main contribution of researchers on "seismic metamaterials" [11, 45] is the exploration of periodic media, the global modal analysis. The "complete mechanical system" consists of the building, its foundations (deep or shallow foundation), and the soil. This approach makes it possible to study and point out interesting effects similar to those already explored in the domain of composite materials at smaller scales in Optics and Crystallography.

\section{Metacity and analogy with urban fabric}

By our observation of certain amalgams of buildings in the urban area (Fig. 6), following the inspirational review article of Wegener's team [29], and ideas put forward by Philippe Guéguen in his visionary work in 2004 [23], where his team observed that seismic noise is reduced or enhanced in cities depending upon the specific arrangement and designs of skyscrapers, we propose here a new way to design urbanism, for self-protecting cities: transformed metacities. The latter are deduced from geometric transformations in the governing equations of elastodynamics that help assemble buildings and streets on transformed city maps, in a way consistent with designs of invisibility cloaks in optics at much smaller scale. What makes this 
work is the fact that using some quasi-conformal grid along which buildings are placed, and consistently with Navier equations of linear elasticity, in the transformed city map, seismic waves would be guided along the paths of minimum length, i.e., geodesics, which would be curved streets or blocks of buildings, according to Fermat's principle of least action. The salient consequence is that some of the seismic waves would be rerouted around most sensitive areas in the city, say power plants, hospitals, stadia, schools, city council, etc. Note also that we could use lakes and underground water tanks, and life lines, etc., to help us design transformed metacities.

The concept of smart cities has been aired for about two decades, a number of geophysicists, notably at University Joseph-Fourier in Grenoble, have already pointed out specific configurations of megalopolis which according to ambient seismic noise display local resonances during earthquakes $[23,42]$. We would like to bridge this concept with the pattern of urban fabric, but with the perspective of side view.

\section{Introduction to quasi/conformal elastodynamics and city maps}

\section{Description of the model}

Following the earlier proposal by Ulf Leonhardt of an invisibility cloak [36] with spatially varying refractive index without anisotropy (conformal optics), we now propose to generate isotropic spatially varying elastic media that greatly simplifies the seismic cloak design. To do this, we need to extend the mathematics of conformal optics to elastodynamic waves. One way to do this is starting from the Navier equations in isotropic elastic medium, to apply the Helmholtz decomposition theorem that splits the displacement field into scalar and vector Lamé potentials that satisfy Helmholtz's equations. One can then apply the coordinate changes to the latter, form invariant, partial differential equations. In 2D, complex analysis allows using conformal mappings which ensure that the transformed parameters remain scalar valued. The propagation of elastic waves is governed by the Navier equations (1):

$\frac{\partial}{\partial x_{i}^{\prime}}\left(C_{i j k l}^{\prime} \frac{\partial}{\partial x_{k}^{\prime}} u_{l}^{\prime}\right)+\rho_{j l}^{\prime} \omega^{2} u_{l}^{\prime}=0$,

where the body force is assumed to be zero, $C=\left(C_{i j k l}\right)$, is the fourth-order elasticity tensor, $\rho^{\prime}=\left(\rho^{\prime}\right)_{j l}$ the secondorder density tensor, $\omega$ is the angular wave frequency, and $u=\left(u_{1}, u_{2}, u_{3}\right)$ is the displacement field. In isotropic homogeneous medium, the solution $u$ of (1) can be decomposed, using the Helmholtz decomposition theorem, into the following equation: $u=\nabla \Phi+\nabla \times \Psi$

with a scalar acoustic potential $\Phi$ and a vector elastic potential $\Psi=\left(\Psi_{1}, \Psi_{2}, \Psi_{3}\right)$ which satisfy the Helmholtz equations (3):

$\left(\Delta+k_{\mathrm{p}}^{2}\right) \Phi=0, \quad\left(\Delta+k_{\mathrm{s}}^{2}\right) \Psi_{q}=0, \quad q \in\{1,2,3\}$,

where the quantities $k_{\mathrm{p}}=\omega / v_{\mathrm{p}}$ and $k_{\mathrm{s}}=\omega / v_{\mathrm{s}}$ are velocities of the dilatational and shear waves, respectively. In terms of the Lamé coefficients $\lambda$ and $\mu$, we can write the equation as follows:

$\left(v_{\mathrm{p}}\right)^{2}=\frac{\lambda+2 \mu}{\rho}, \quad\left(v_{\mathrm{s}}\right)^{2}=\frac{\mu}{\rho}$.

In conformal cloaking, one introduces a conformal (plane) transformation $f(x, y)$ having components $\left(f_{1}(x, y), f_{2}(x, y)\right)$. Thanks to Cauchy-Riemann conditions, we write (5):

$\frac{\partial f_{1}}{\partial x}=\frac{\partial f_{2}}{\partial y}$ and $\frac{\partial f_{2}}{\partial x}=-\frac{\partial f_{1}}{\partial y}$.

Under these conditions, Eq. (3) retains their form. Namely, their transformed equations read

$$
\left(\Delta^{\prime}+k_{\mathrm{p}}^{\prime 2}\right) \Phi^{\prime}=0, \quad\left(\Delta^{\prime}+k_{\mathrm{s}}^{\prime 2}\right) \Psi_{q}^{\prime}=0, \quad q \in\{1,2,3\},
$$

where $\Delta^{\prime}$ is the Laplacian in the transformed coordinates and $k_{\mathrm{p}}^{\prime}=\omega / v_{\mathrm{p}}^{\prime}, k_{\mathrm{s}}^{\prime}=\omega / v_{\mathrm{s}}^{\prime}$, with

$\left(v_{\mathrm{p}}^{\prime}\right)^{2}=\frac{\lambda^{\prime}+2 \mu^{\prime}}{\rho^{\prime}}, \quad\left(v_{\mathrm{s}}^{\prime}\right)^{2}=\frac{\mu^{\prime}}{\rho^{\prime}}$.

The transformed Lamé parameters $\lambda^{\prime}, \mu^{\prime}$ and density $\rho^{\prime}$ are spatially dependent, as they are related to the transformation's Jacobian. Therefore, the resulting transformed elastic medium is isotropic with a symmetric elasticity tensor, which is inhomogeneous. Thus, a further engineering process is needed to obtain a heterogeneous medium with desired properties.

\section{Concept of quasi/conformal grid for transformed metacities with carpet cloaks}

Since we are now on safe ground with the governing equations of linear elastodynamics obeying precise transformational rules, we can start designing cities of the future using the concept of quasi-conformal city maps. Following the advent of conformal optics, a broadband plasmonic carpet has been designed and realized with small pillars (about $200 \mathrm{~nm}$ in diameter and height) placed at the nodes of a quasi-conformal map thanks to nanotechnology techniques on a metallic surface [43]. This carpet cloak 
operates for electromagnetic wave wavelengths from 650 to $950 \mathrm{~nm}$ (from visible to near-infrared light). In what follows, we follow the same route towards design of a carpet cloak for surface Rayleigh waves, scaling up the plasmonic cloak design by a factor of one hundred thousand. This can be done thanks to the similarity of transformed elasticity Eqs. (6) and (7) with transformed plasmonic equations in [43].

In Fig. 7, we illustrate the first step of the design process towards transformed metacity through a conformal map. Following $\mathrm{Li}$ and Pendry's proposal of an invisibility carpet with a quasi-conformal grid to minimize artificial anisotropy [27], we consider a square network of streets and buildings (Fig. 7-upper panel) which we map it onto a quasi-conformal network of streets with square buildings (Fig. 7-lower panel). One can note that curved streets meet at a right angle (which ensures the weak anisotropy of the carpet cloak) and buildings of square cross section have sides parallel to these streets.

In Fig. 8, we illustrate the step 2 in the design of a transformed metacity through a conformal map. Here, we consider a more realistic city map along a sea shore (upper
Fig. 7 Concept of quasiconformal transformed city map (sky view)
Fig. 8 Concept of quasiconformal transformed city map (sky view)
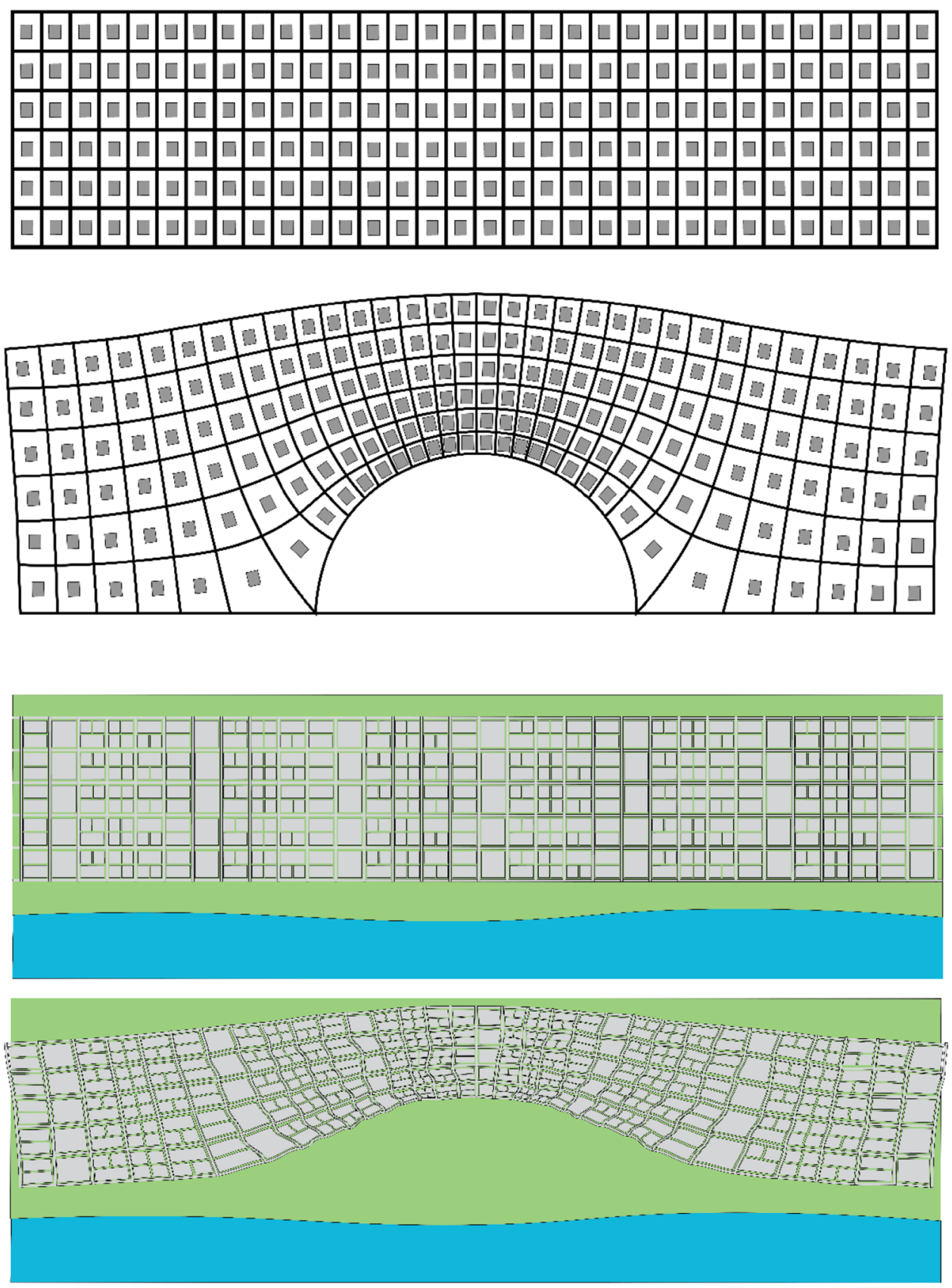
Fig. 9 Concept of quasiconformal transformed city map (3D view). Final result of designing a metacity via conformal mapping
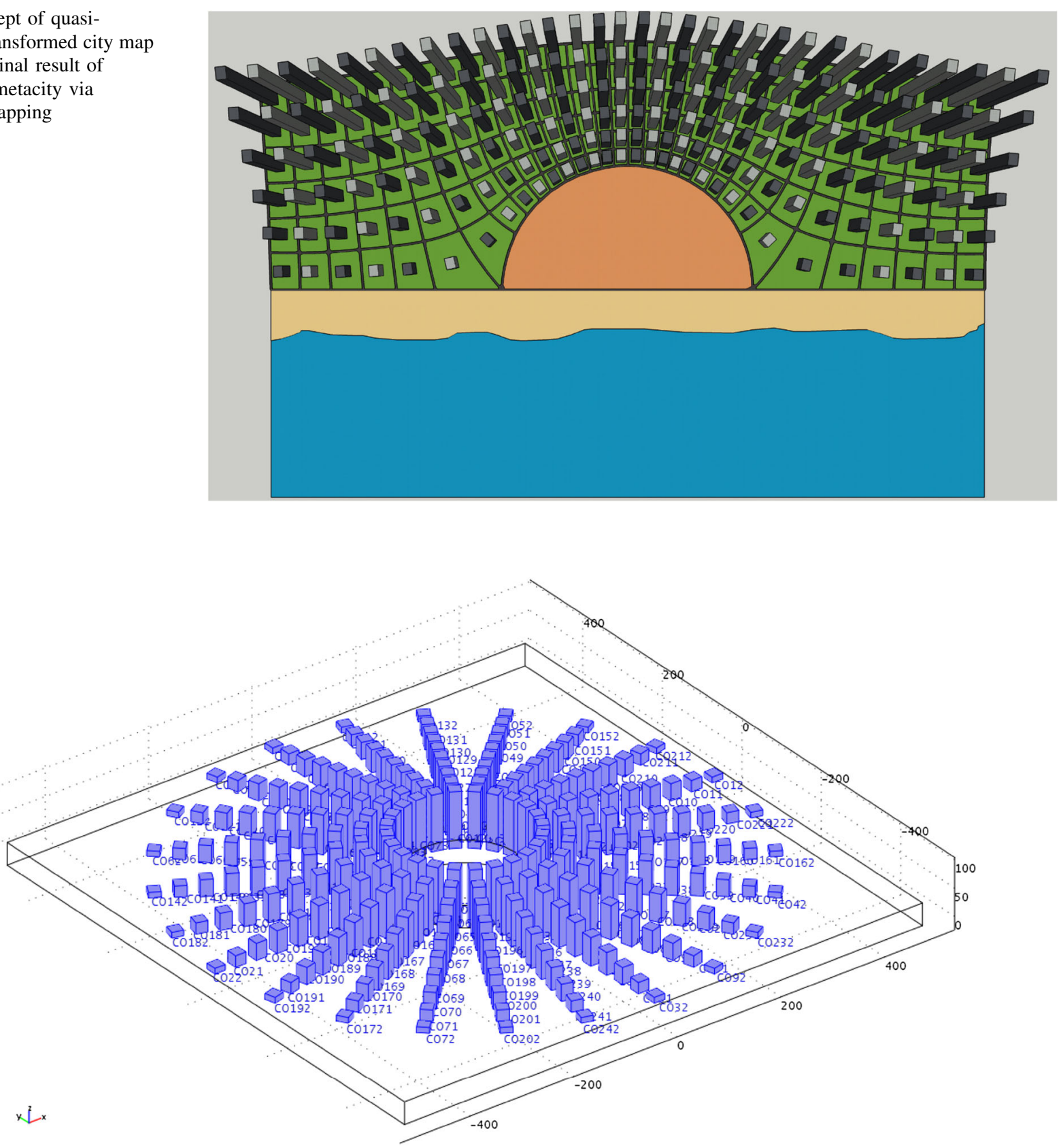

Fig. 10 Concept of quasi-conformal transformed city map (sky view). Illustration of the first step in the design of a metacity through a conformal map for an invisibility cloak like in [36]. The distribution of buildings (made of concrete) within this seismic cloak which is

panel), which is then conformally mapped onto a transformed city map, with buildings of trapezoidal cross section. Such a design might be difficult to implement in practice, see Fig. 8 for an alternative. The illustration of step 3 in the design of a simplified transformed metacity through a conformal map is presented in Fig. 9. One can now see the final result of designing a metacity via conformal mapping.
$1 \mathrm{~km}$ in diameter, with building's height ranging from 10 to $100 \mathrm{~m}$, which are partially buried in the soil (with a depth of $40 \mathrm{~m}$ ), mimics the spatially varying refractive index in a conformal invisibility cloak

\section{Concept of quasi/conformal grid for transformed metacities with invisibility cloaks}

Thus far, we have used quasi-conformal grids following the lead of Li and Pendry, by taking the carpet cloaks which they originally designed for control of light at the nanoscale and implementing them for cities located near a sea shore (or a mountain) at the meter scale. We would now 



Fig. 11 Numerical simulation (Comsol Multiphysics) for the proof of concept of the seismic cloak of Fig. 10. Representative snapshots (increasing time steps clockwise from upper left to lower left panel) for a Rayleigh wave coming from the left at frequency $0.5 \mathrm{~Hz}$ (color scale with arbitrary unit of energy)

like to propose a city which has self-protection features against earthquakes coming from all directions. Here, we use some analogies with the seminal work of Ulf Leonhardt, who proposed a route towards invisibility cloaks using some spatially varying (but isotropic) material parameters [36]. We show in Fig. 10 the design of a seismic cloak, which is $1 \mathrm{~km}$ in diameter and whose buildings have increasing height towards the center, just like the refractive index in Leonhardt's optical cloak. The region in the center of the metacity (e.g., a park) is protected against Rayleigh waves, as demonstrated by the numerical simulations in Comsol Multiphysics for an incoming wave of $0.5 \mathrm{~Hz}$, see Fig. 11. Here, we do not take into account the pounding of buildings, because they are far apart.

\section{Discussion and perspectives}

In this article, we put forward the new concept of transformational urbanism and metacities.

The novelty of this paper is the introduction of the dynamic response of a megastructure, as a whole district rather than as distinct individual buildings.

These new tools for Architects may lead in the future to another way of thinking about structuring cities and thence the urban fabric. Indeed, a major point is that this challenges the "passive-approach" of seismic design, i.e., where the building has to be adapted to the "imposed" seismic input, into an approach where a group of structured buildings, capable of reacting together, at the district scale in the event of seismic disturbance, are considered together.

To conclude, we comment that there are yet further potential analogies between electromagnetic metamaterials displaying artificial magnetism, so-called split-ring resonators introduced by Sir John Pendry in the late 1990s $[37,39]$, and metacities with buildings or building blocks displaying a cross section like split-ring resonators, see Fig. 12. The subwavelength nature of the frequency stop

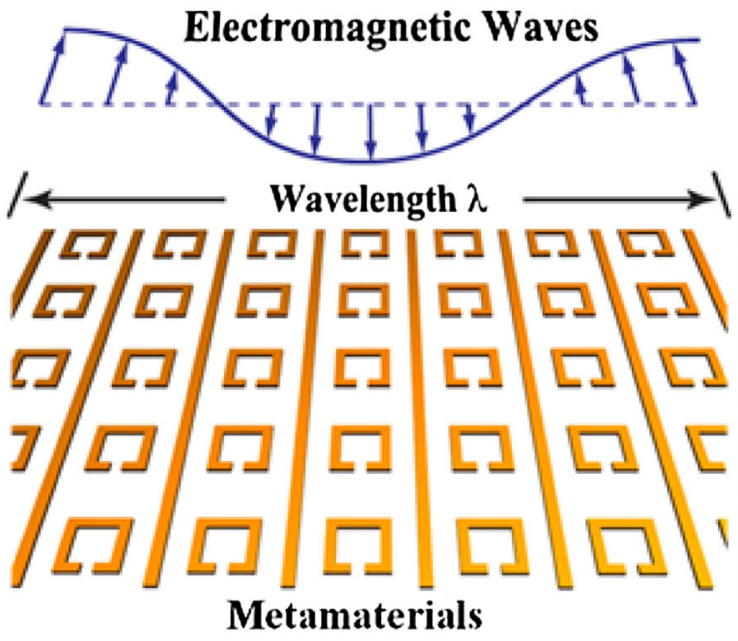

Fig. 12 Analogies between electromagnetic (nano-scale) metamaterial and (decameter scale) metacity. Pendry's split-ring resonators for artificial magnetism and negative refractive Index (left panel) bear some resemblance with a metacity viewed from sky (right panel).
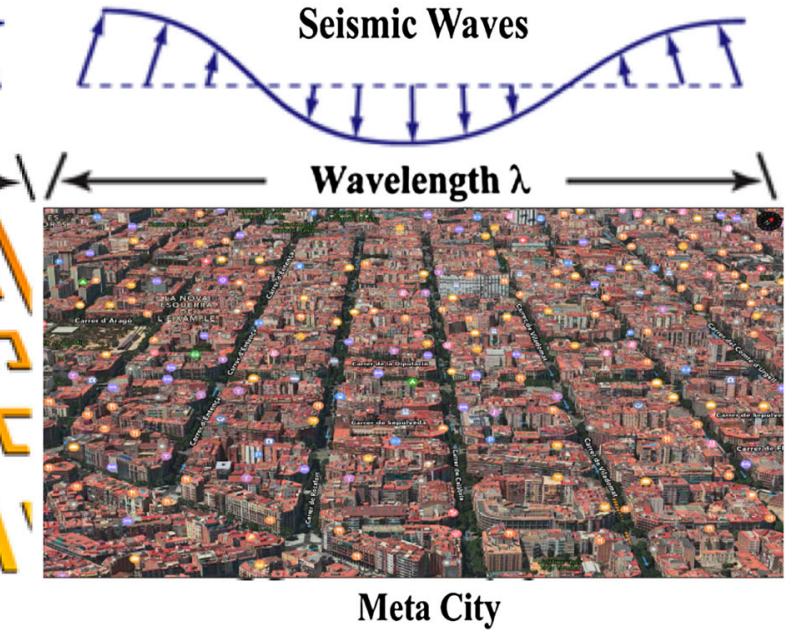

Subwavelength local resonances of buildings shaped as split-ring resonators create low-frequency stop bands which can be used to reflect seismic waves 
band responsible for magnetic activity in electromagnetic metamaterials can be used as a seismic shield via lowfrequency elastic stop band in the metacity.

For seismic waves interacting with a structured surface, that is a city, it is essential to recognise that established concepts from optics and electromagnetic waves, used there to design and create desired behaviour, can be adapted or interpreted to the seismic context. Our purpose here has been to highlight the main concepts and analogies and implement them into the seismic context thereby to motivate and inspire future studies.

\section{References}

1. Achaoui Y, Ungureanu B, Enoch S, Brûlé S, Guenneau S (2016) Seismic waves damping with arrays of inertial resonators. Extreme Mech Lett, 8:30-37

2. Auriault JL, Boutin C, Geindreau C (2009) Homogénéisation de phénomènes couplés en milieux hétérogènes, Mécanique et Ingénierie des Matériaux, Hermes, Lavoisier

3. Aznavourian R, Puvirajesinghe T, Brûlé S, Enoch S, Guenneau S (2016) Bio-inspired seismic metamaterials with transformed elastic crystals. J Phys Condens Matter (provisionally accepted)

4. Bielak J (1975) Dynamic behaviour of structures with embedded foundations. Earthq Eng Struct Dyn 3:259-274

5. Boutin C, Roussillon P (2004) Assessment of the urbanization effect on seismic response. Bull Seismol Soc Am 94(1):251-268

6. Boutin C, Roussillon P (2006) Wave propagation in presence of oscillators on the free surface. Int J Eng Sci 4:180-204

7. Brûlé S, Javelaud EH, Enoch S, Guenneau S (2017a) Flat lens for seismic waves. Sci Rep (in progress)

8. Brûlé S, Enoch S, Guenneau S, Craster RV (2017b) Seismic metamaterials: controlling surface Rayleigh waves using analogies with electromagnetic metamaterials. In: Handbook of metamaterials. World Scientific (in press)

9. Brûlé S, Duquesnoy S (2016) Change of ground type by means of dynamic compaction: consequences on the calculation of seismic loadings. Innov Infrastruct Solut 1:39. doi:10.1007/s41062-0160037-4

10. Brûlé S, Enoch S, Guenneau S (2017). Structured soils under dynamic loading: The metamaterials in Geotechnics. Revue Française de Géotechnique (in press)

11. Brûlé S, Javelaud EH, Enoch S, Guenneau S (2014) Experiments on seismic metamaterials: molding surface waves. Phys Rev Lett 112:133901

12. Clouteau D, Aubry D (2001) Modification of the ground motion in dense urban areas. J Comput Acoust 9(4):1659-1675

13. Colombi A, Roux P, Guenneau S, Rupin M (2015) Directional cloaking of flexural waves in a plate with a locally resonant metamaterial. J Acoust Soc Am 137(4):1783-1789

14. Colombi A, Roux P, Guenneau S, Guéguen P, Craster RV (2016) Forests as a natural seismic metamaterial: Rayleigh wave bandgaps induced by local resonances. Sci Rep 6:19238

15. Diatta A, Guenneau S (2014) Controlling solid elastic waves with spherical cloaks. Appl Phys Lett 105(2):021901

16. Diatta A, Achaoui Y, Brûlé S, Enoch S, Guenneau S (2016) Control of Rayleigh-like waves in thick plate Willis metamaterials. AIP Adv 6(12):121707

17. Dupont G, Kimmoun O, Molin B, Guenneau S, Enoch S (2015) Numerical and experimental study of an invisibility carpet in a water channel. Phys Rev E 91:023010
18. Farhat M, Enoch S, Guenneau S, Movchan AB (2008) Broadband cylindrical acoustic cloak for linear surface waves in a fluid. Phys Rev Lett 101:1345011

19. Farhat M, Guenneau S, Enoch S, Movchan AB (2009) Cloaking bending waves propagating in thin elastic plates. Phys Rev B 79(3):033102

20. Guéguen P, Bard PY, Semblat JF (2000) From soil-structure interaction to site-city interaction. In: 12th World conference on earthquake engineering, Auckland, New Zealand

21. Guéguen Ph, Bard P-Y, Chàvez-Garcia FJ (2002) Site-city seismic interaction in Mexico City-like environments: an analytical study. Bull Seismol Soc Am 92(2):794-811

22. Ghergu M, Ionescu IR (2009) Structure-soil-structure coupling in seismic excitation and city effect. Int J Eng Sci 47:342-354

23. Guillier B, Machane D, Oubaiche EH, Chatelain J-L, Ait Meziane Y, Ben Salem R Dunand F,Guéguen P, Hadid M, Hellel M, Kiboua A, Laouami N, Mezouer N, Nour A, Remas A (2004) Résultats préliminaires sur les fréquences fondamentales et les amplifications de sols, obtenus par l'étude du bruit de fond, sur la ville de boumerdes-algérie. Mém Serv. Géol. Alg. 12:103-114

24. Housner GW (1954) Effect of foundation compliance on earthquake stresses in multistory buildings. Bull Seismol Soc Am 44:551-569

25. Housner GW (1957) Interaction of building and ground during an earthquake. Bull Seismol Soc Am 47:179-186

26. Hu X, Chan CT (2005) Refraction of water waves by periodic cylinder arrays. Phys Rev Lett 95:154501

27. Li J, Pendry JB (2008) Hiding under the carpet: a new strategy for cloaking. Phys Rev Lett 101(20):203901

28. Iorch IV, Muskhin IS, Shadrivov IV, Belov PA, Kivshar YS (2013) Hyperbolic metamaterials based on multilayer graphene structures. Phys Rev B 87:075416-6

29. Kadic M, Bückmann T, Schittny R, Wegener M (2013) Metamaterials beyond electromagnetism. Rep Prog Phys 76:26501

30. Kim SH, Das MP (2012) Seismic waveguide of metamaterials. Mod Phys Lett B 26:1250105

31. Krodel S, Thome N, Daraio C (2015) Wide band-gap seismic metastructures, Ex Mech Letters 4:111-117

32. Kushwaha MS, Halevi P, Dobrzynski L, Djafari-Rouhani B (1993) Accoustic band structure of periodic elastic composites. Phys Rev Lett 71:2022-2025

33. Kushwaha MS (1997) Stop-bands for periodic metallic rodssculptures that can filter the noise. Appl Phys Lett 70(24):3218-3220

34. Lakes R (1987) Foam structures with a negative Poisson's ratio. Science 235(4792):1038-1040

35. Ledoux CN (1804) L'Architecture considérée sous le rapport de l'Art, des Mœurs et de la Législation, Tome 1, Imprimerie Perronneau, Paris

36. Leonhardt U (2006) Optical conformal mapping. Science 312(5781):1777-1780

37. Ramakrishna SA (2005) Physics of negative refractive index materials. Rep Prog Phys 68(2):449

38. Sánchez-Pérez JV, Caballero D, Mártinez-Sala R, Rubio C, Sánchez-Dehesa J, Meseguer F, Llinares J, Gálvez F (1998) Sound attenuation by a two-dimensional array of rigid cylinders. Phys Rev Lett 80:5325

39. Pendry JB, Holden AJ, Robbins DJ, Stewart WJ (1999) Magnetism from conductors and enhanced nonlinear phenomena. IEEE Trans Microw Theory Tech 47(11):2075-2084

40. Pendry JB (2000) Negative refraction makes a perfect lens. Phys Rev Lett 85:3966-3969

41. Pendry JB, Schurig D, Smith DR (2006) Controlling electromagnetic fields. Science 312(5781):1780-1782

42. Perrault M, Guéguen P (2015) Correlation between ground motion and building response using Californian earthquake records. Earthq Spectra 31(4):2027-2046 
43. Renger J, Kadic M, Dupont G, Aćimović SS, Guenneau S, Quidant R, Enoch S (2010) Hidden progress: broadband plasmonic invisibility. Opt Express 18(15):15757-15768

44. Semblat JF, Pecker A (2009) Waves and vibrations in soils: earthquakes, traffic, shocks, construction works. IUSS Press, Pavia

45. Sheng P (2014) Viewpoint: a step towards a seismic cloak. Physics 7:34

46. Spiliopoulos KV, Anagnospoulos SA (1992) Earthquake induced pounding in adjacent building. In: Earthquake engineering, 10th World conference, 1992, Balkema, Rotterdam

47. Trifunac MD (1972) Interaction of a shear wall with the soil for incident plane SH waves. Bull Seismol Soc Am 62:63-83
48. Ungureanu B, Achaoui Y, Enoch S, Brûlé S, Guenneau S (2016) Auxetic-like metamaterials as novel earthquake protections. EPJ Appl Metamat 2015(2):17

49. Walser RM (2001) Electromagnetic metamaterials. In: Paper presented at the International Society for Optical Engineering (SPIE), San Diego, USA, 4467, 1-165

50. Wirgin A, Bard P-Y (1996) Effects of buildings on the duration and amplitude of ground motion in Mexico City. Bull Seismol Soc Am 86(3):914-920

51. Wong HL, Trifunac MD, Westermo B (1977) Effects of surface and subsurface irregularities on the amplitude of monochromatic waves. Bull Seismol Soc Am 67:353-368 